\title{
Clinical Profile, Bacterial Isolates and Antibiotic Susceptibility Patterns in Urinary Tract Infection in Children - Hospital Based Study
}

\author{
Malla KK ${ }^{1}$, Sarma MS ${ }^{2}$, Malla $\mathbf{T}^{3}$, Thapalial $\mathbf{A}^{4}$ \\ ${ }^{1}$ Dr. Kalpana Karmacharya Malla, Associate Professor, ${ }^{2}$ Dr. M.S. Sarma, Assistant Professor, ${ }^{3}$ Dr Tejesh Malla, \\ Assistant Professor, ${ }^{4}$ Prof Anna Thapalial, Professor and Head of Department, Department of Pediatrics, Manipal \\ College of Medical Sciences and Teaching Hospital, Pokhara, Nepal
}

Address for Correspondence: Dr. Kalpana K Malla. E-mail: kalpana17@hotmail.com

\begin{abstract}
Background: The present study analyzes the clinical profile and identifies the pathogenic distribution and their antimicrobial susceptibility pattern in childhood urinary tract infections in order to provide standard reference for optimal use of antibiotics in Nepal. Materials and Methods: Prospective study conducted in the paediatric unit, Manipal College of Medical Sciences and Teaching Hospital, Pokhara over a period of 18 months. A total of 168 patients of both sexes, ranging from post neonatal period to fifteen years of age were studied. The modes of presentation, laboratory investigation reports which included urine routine microscopy, bacterial isolates with colony count from the urine culture and antibiotic sensitivity pattern were documented. Result: Urinary tract infection was common among females $(67.2 \%)$ with female to male ratio $2: 1$. Urine culture was positive in $57 \%$ of cases. Escherichia coli was the most common organism isolated (50\%), followed by Klebsiella (16.66\%), Proteus (12.50\%), Enterococcus (6.25\%). Among the Aminoglycosides, Amikacin was found to have the highest sensitivity (96\%) amongst most bacteria. Netilimycin and Gentamycin had sensitivity of $70.8 \%$ and $64.86 \%$ respectively. Favourable sensitivity was seen with Nitrofurantoin (88.88\%), Norfloxacin (78.3\%), Ciprofloxacin $(80.9 \%)$. Though sensitivity to Vancomycin was tested only in 14 cases it was found that Vancomycin was 100\% sensitive to Staphylococcus, Enterococcus, E.coli and Klebsiella. Highest degree of resistance was noticed with Amoxicillin (92\%), Ampicillin (85.36\%), Ceftazidime (85.71\%), Amoxyclav (83.33\%) and Cefotaxime (76.47\%), Cefazolin (62.85\%), Ofloxacin(66.66\%) and Ceftriaxone (66.66\%). Conclusion: Infected urine stimulates an immunological and inflammatory response leading to renal injury and scarring, ultimately leading to end stage renal failure. In a subtropical country like ours, there is a temporal relationship in the antibiotic sensitivity pattern of UTI. Hence frequent large scale studies are required from time to time to note the changes in sensitivity and resistance. Complicated UTI and subsequent renal failure still continues to be one of the major causes of mortality in children in this part of Asia. From this study it can be concluded that E.coli still remains as the commonest isolate in UTI. Aminoglycosides and Fluoroquinolones (except Ofloxacin) can be considered as first line drugs. An emerging resistance has been noticed with Cephalosporins. Vancomycin may be a reserve drug of choice in failed or mutidrug resistant cases. Large-scale multicentre studies are required to generalise the data for the whole country.
\end{abstract}

Key words: Urinary tract infection, culture and susceptibility patterns

\section{Introduction}

Urinary tract infection (UTI) is common in paediatric practice and an important cause of morbidity and mortality in children. Though UTI is a common problem throughout the world the microbial isolates and their sensitivity pattern needs to be analysed at intervals to monitor the changing patterns of microbial flora and the development of resistance to drugs which may help physicians to treat UTI in a better way and to prevent further complications. As far as our knowledge is concerned, this study is unique as it is among the first of its kind to be conducted and published amongst the paediatric age group in Nepal. 


\section{Materials and Methods}

We conducted a prospective analysis of the cases attending paediatric OPD and those admitted in the ward of Manipal Teaching Hospital, Pokhara, Nepal. Study period was for 18 months from the $1^{\text {st }}$ July 2005 to $31^{\text {st }}$ December 2007. Children of both sexes upto the age of 15 years were included in this study. Neonates were excluded. Their clinical presentations with associated conditions and risk factors were noted. Approximately 500 children were initially screened but only 168 children were analysed for the study as they fulfilled our inclusion criteria listed below. Parents were explained about the study and professional care was taken to collect the urine sample for culture and sensitivity by sterile technique. Urine was sampled for culture by aseptic supra-pubic bladder aspiration in infants. Sterile plastic receptacles were used for collection of urine in younger patients to avoid contamination with stools. Clean catch mid stream urine was sampled in older children and adolescents after proper cleansing of urethra and under supervision. The samples were then processed for routine microscopy. Only samples with more than $5 \mathrm{WBC}$ per high power field (hpf) were subjected for culture and antimicrobial susceptibility testing in the bacteriology laboratory of Manipal Teaching Hospital.

\section{Inclusion Criteria}

1. Fever and/or acute voiding symptoms (as shown in Table 2) between ages of $2^{\text {nd }}$ month of life to 15 years.

2. Only children with WBC count more than $5 /$ hpf in centrifuged freshly voided urine.

\section{Exclusion Criteria}

1. Neonates.

2. Children with pre-existing renal diseases like VUR and PUV.

3. Acute coexisting renal diseases like Glomerulonephritis and Nephrotic Syndrome.

4. Recurrent UTI.

5. Previous antibiotic usage.

\section{Significant culture/ culture positive}

1. Receptacle samples and mid stream urine sample with cultures with $>10^{5}$ colony forming units of bacteria/ml of urine in young infants and adolescents.

2. Any colony count with suprapubic aspiration in infants.

\section{Observation and Results}

In the 168 children (Female: Male $=2: 1$ ) fulfilling the inclusion criteria, $96(57 \%)$ had a positive culture in urine. The rest $72(42.9 \%)$ were either culture negative or they had colony count $<10^{5}$ (Fig 1).
Table 1: Age and Sex Distribution at Presentation $(\mathrm{N}=168)$

\begin{tabular}{|l|c|c|c|c|}
\hline \multirow{2}{*}{ SEX } & \multicolumn{4}{|c|}{ AGE } \\
\cline { 2 - 5 } & $<\mathbf{1 Y R}$ & $\mathbf{> 1 - 5 Y R}$ & $\mathbf{6 - 1 0 Y R}$ & $>\mathbf{1 0 Y R}$ \\
\hline $\begin{array}{l}\text { MALE }=\mathbf{5 5} \\
(\mathbf{3 2 . 7} \%)\end{array}$ & 15 & 20 & 10 & 10 \\
\hline $\begin{array}{l}\text { FEMALE = 113 } \\
(\mathbf{6 7 . 2 \% )}\end{array}$ & 19 & 40 & 34 & 20 \\
\hline TOTAL & 34 & 60 & 44 & 30 \\
\hline
\end{tabular}

Fig. 1: Urine Culture Report

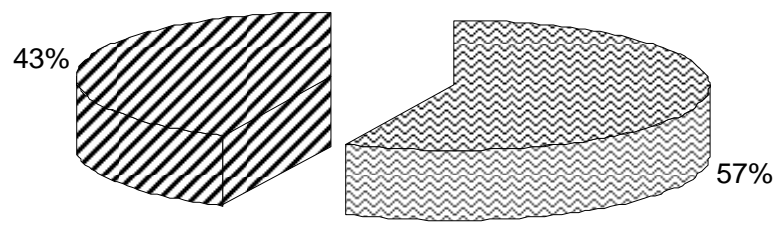

\&Culture positive UTI $\square$ Culture negative UTI

The common clinical presentations were fever (86.9\%), pain abdomen (46.4\%) and vomiting (39.28\%). Other symptoms included chills, rigors, dysuria, burning micturition, increased urine frequency, hematuria, oliguria, facial puffiness, vaginal discharge, loose stools, febrile seizures, concomitant respiratory tract infection, constipation, hepatosplenomegaly, irritablity (Table 2).

Table 2: Signs and Symptoms at Presentation

\begin{tabular}{|l|c|c|}
\hline Clinical Picture & $\begin{array}{c}\text { Number } \\
(\mathbf{n = 1 6 8 )}\end{array}$ & Percentage \\
\hline Fever & 146 & $86.9 \%$ \\
\hline Pain Abdomen & 78 & $46.4 \%$ \\
\hline Vomiting & 66 & $39.28 \%$ \\
\hline Dysuria & 40 & $23.8 \%$ \\
\hline Chills and Rigors & 30 & $17.8 \%$ \\
\hline Burning Micturition & 26 & $15.4 \%$ \\
\hline Loose Stool & 24 & $14.28 \%$ \\
\hline Increased Frequency & 22 & $13.0 \%$ \\
\hline Febrile Seizures & 22 & $13.0 \%$ \\
\hline Concomitant & & \\
Respiratory tract & 22 & $13.0 \%$ \\
\hline Infection & & \\
\hline Haematuria & 18 & $10.7 \%$ \\
\hline Facial puffiness & 12 & $7.1 \%$ \\
\hline Hepatosplenomegaly & 12 & $7.1 \%$ \\
\hline Constipation & 12 & $7.1 \%$ \\
\hline
\end{tabular}




\begin{tabular}{|l|c|c|}
\hline Oliguria & 12 & $7.1 \%$ \\
\hline Vaginal discharge & 10 & $5.95 \%$ \\
\hline Irritability & 10 & $5.95 \%$ \\
\hline Failure to Thrive & 10 & $5.95 \%$ \\
\hline Decreased Appetite & 6 & $3.57 \%$ \\
\hline Bed Wetting & 4 & $2.38 \%$ \\
\hline Phimosis & 4 & $2.38 \%$ \\
\hline $\begin{array}{l}\text { Recurrent Pain } \\
\text { Abdomen }\end{array}$ & 4 & $2.38 \%$ \\
\hline Encopresis & 1 & $0.59 \%$ \\
\hline
\end{tabular}

The most common organism isolated was E.coli (50\%) followed by Klebsiella pneumoniae (16.66\%), Proteus vulgaris (12.50\%), Enterococcus (6.25\%). Citrobacter species, Enterobacter and Staphylococcus aureus were isolated in $4.10 \%$ each. Pseudomonas aerogenosa was isolated in only $2.08 \%$ of cases. (Fig 2). No anaerobic organism or fungemia was reported.

Among the Aminoglycosides, Amikacin had the highest sensitivity (96\%) while $4.16 \%$ of Enterococcus and Pseudomonas were resistant. Netilimycin and Gentamycin had sensitivity of $70.8 \%$ and $64.86 \%$ respectively. The three organisms totally resistant to Gentmicin were Citrobacter, Enterobacter and Staphylococcus. Ciprofloxacin and Norfloxacin were found to be sensitive in almost all bacteria except Pseudomonas which was $100 \%$ resistance to Norfloxacin. Nalidixic acid and Co-trimoxazole showed variable resistance amongst the organisms isolated. Sensitivity for Vancomycin (100\%), Cephalexin (50\%), Ofloxacin (33.33\%) and
Ceftriaxone (33.33\%) was tested only in 12 cases. Among them Vancomycin was found to be $100 \%$ sensitive to Enterococcus, Staphylococcus, E.coli and Klebsiella.Highest degree of resistance was noticed with Amoxicillin (92\%), Ampicillin (85.36\%), Ceftazidime (85.71\%), Amoxy-clav (83.33\%), Cefotaxime (76.47\%), Cefazolin (62.85\%), Ofloxacin (66.66\%) and Ceftriaxone(66.66\%).

The sensitivity pattern of various organisms was also studied. E.coli responded better with Aminoglycosides, Fluoroquinolones and Nitrofurantoin but displayed a high resistance to most of the Cephalosporins. Unfortunately sensitivity with fourth generation Cefepime was not tested in any cases. Resistance was also noticed with Ampicillin, Amoxicillin and Amoxyclav. The sensitivity pattern of Klebsiella and Proteus was similar with few minor differences like Proteus showing higher sensitivity pattern towards Norfloxacin (50\%), Nitrofurantoin (33.33\%), Co-trimoxazole $(41.66 \%)$ as compared to Klebsiella showing sensitivity to Norfloxacin $(31.25 \%)$ Nitrofurantoin(12.5\%) and Co-trimoxazole (18.75\%). Amongst the gram negative bacteria studied only Enterococcus was $100 \%$ resistant to Amikacin. Though almost all organisms showed $80-100 \%$ resistance to penicillins, Enterococcus was $100 \%$ sensitive to amoxy-clav. Majority of the Cephalosporins were ineffective to the bacteria isolated. Majority of the bacteria except Enterobacter was sensitive to Nitrafurantoin. Citrobacter, Enterobacter and Staphylococcus showed some response to most Aminoglycosides except Gentamycin. Vancomycin showed no resistance but the sensitivity for Vancomycin was tested only in 14 cases $(14.5 \%)$.

Fig. 2: Bacterial isolates of culture positive cases

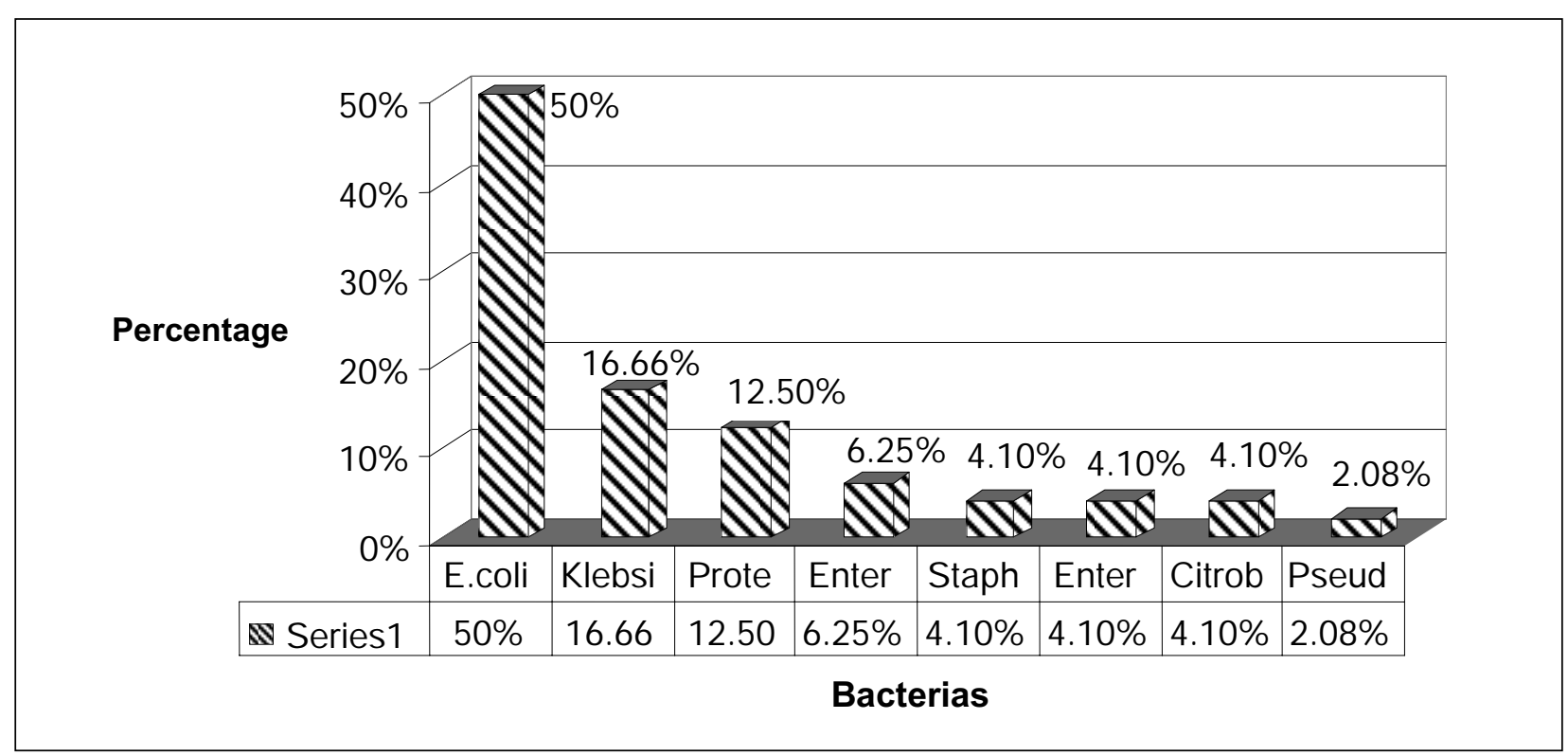


Table 3: Antibiogram of Culture Positive Cases (N=96)

\begin{tabular}{|c|c|c|c|c|c|c|c|c|}
\hline \multirow{2}{*}{ Antibiotic } & \multirow{2}{*}{$\begin{array}{c}\text { Sensitivity } \\
\text { Tested }\end{array}$} & \multirow{2}{*}{$\begin{array}{l}\text { Sensitivity } \\
\text { Not Tested }\end{array}$} & \multicolumn{2}{|c|}{ Sensitive } & \multicolumn{2}{|c|}{$\begin{array}{l}\text { Intermediate } \\
\text { Sensitive }\end{array}$} & \multicolumn{2}{|c|}{ Resistant } \\
\hline & & & No & $\%$ & No & $\%$ & No. & $\%$ \\
\hline Amikacin & 50 & 46 & 48 & 96.0 & 0 & 0 & 2 & 4.2 \\
\hline Netilimycin & 48 & 48 & 34 & 70.8 & 4 & 8.3 & 10 & 20.8 \\
\hline Gentamycin & 74 & 22 & 48 & 64.9 & 0 & 0 & 26 & 35.1 \\
\hline Amoxy-clav & 36 & 60 & 6 & 16.7 & 0 & 0 & 30 & 83.3 \\
\hline Ampicillin & 82 & 14 & 12 & 14.6 & 0 & 0 & 70 & 85.4 \\
\hline Amoxycillin & 50 & 46 & 4 & 8.0 & 0 & 0 & 46 & 92.0 \\
\hline Cefotaxime & 34 & 62 & 8 & 23.5 & 0 & 0 & 26 & 76.5 \\
\hline Cefazoline & 70 & 26 & 24 & 34.3 & 2 & 2.9 & 44 & 62.8 \\
\hline Ceftazidime & 14 & 82 & 2 & 14.3 & 0 & 0 & 1 & 85.7 \\
\hline Ceftriaxone & 12 & 84 & 4 & 33.3 & 0 & 0 & 8 & 66.7 \\
\hline Cephalexin & 12 & 84 & 6 & 50.0 & 0 & 0 & 6 & 50.0 \\
\hline Ciprofloxacin & 42 & 54 & 34 & 80.9 & 2 & 4.8 & 6 & 14.3 \\
\hline Nalidixic acid & 32 & 64 & 14 & 43.8 & 0 & 0 & 18 & 56.2 \\
\hline Norfloxacin & 74 & 22 & 58 & 78.3 & 0 & 0 & 16 & 21.6 \\
\hline Co-trimoxazole & 66 & 30 & 3 & 57.6 & 0 & 0 & 2 & 42.4 \\
\hline Nitrofurantoin & 54 & 42 & 48 & 88.9 & 0 & 0 & 6 & 11.1 \\
\hline Vancomycin & 14 & 82 & 14 & 100.0 & 0 & 0 & 0 & 0 \\
\hline Ofloxacin & 12 & 84 & 4 & 33.3 & 0 & 0 & 8 & 66.7 \\
\hline
\end{tabular}


Table 4a: The Antibiotic Sensitivity of the Organisms Isolated in all the Cases.

\begin{tabular}{|c|c|c|c|c|c|c|c|c|}
\hline & \multicolumn{8}{|c|}{ Organisms (\% sensitive) } \\
\hline Drugs & 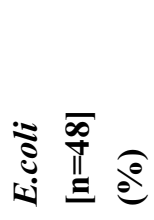 & 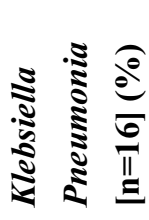 & 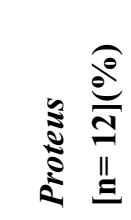 & 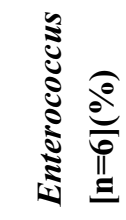 & 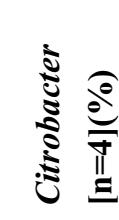 & 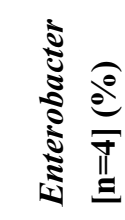 & 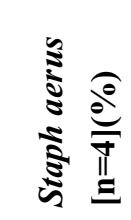 & 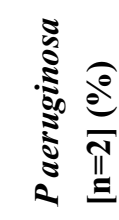 \\
\hline $\begin{array}{l}\text { Amikacin } \\
(\mathrm{n}=\mathbf{5 0}) \\
\mathrm{IS}=\mathbf{0}\end{array}$ & $\begin{array}{l}S=24 \\
100 \% \\
R=0\end{array}$ & $\begin{array}{l}S=8 \\
100 \% \\
R=0\end{array}$ & $\begin{array}{l}S=6 \\
100 \% \\
R=0\end{array}$ & $\begin{array}{l}S=0 \\
R=2 \\
100 \%\end{array}$ & $\begin{array}{l}S=4 \\
100 \% \\
R=0\end{array}$ & $\begin{array}{l}S=4 \\
100 \% \\
R=0\end{array}$ & $\begin{array}{l}S=2 \\
100 \% \\
R=0\end{array}$ & ND \\
\hline $\begin{array}{l}\text { Netlimycin } \\
(n=48)\end{array}$ & $\begin{array}{l}S=18 \\
\mathbf{6 9 . 2 \%} \\
R=4 \\
15.38 \% \\
I S=4 \\
15.38 \%\end{array}$ & $\begin{array}{l}S=4 \\
100 \% \\
R=0 \\
I S=0\end{array}$ & $\begin{array}{l}S=4 \\
\mathbf{6 6 . 6 6 \%} \\
R=2 \\
33.33 \% \\
I S=0\end{array}$ & ND & $\begin{array}{l}S=2 \\
50 \% \\
R=2 \\
50 \% \\
I S=0\end{array}$ & $\begin{array}{l}S=2 \\
100 \% \\
R=0 \\
I S=0\end{array}$ & $\begin{array}{l}S=0 \\
R=2 \\
100 \% \\
I S=0\end{array}$ & $\begin{array}{l}S=2 \\
100 \% \\
R=0 \\
I S=0\end{array}$ \\
\hline $\begin{array}{l}\text { Gentamycin } \\
(\mathrm{n}=74) \\
\mathrm{IS}=\mathbf{0}\end{array}$ & $\begin{array}{l}S=26 \\
\mathbf{7 2 . 2 2 \%} \\
R=10 \\
27.77 \%\end{array}$ & $\begin{array}{l}S=8 \\
\mathbf{6 6 . 6 6 \%} \\
R=4 \\
33.33 \%\end{array}$ & $\begin{array}{l}S=8 \\
\mathbf{8 0} \% \\
R=2 \\
20 \%\end{array}$ & $\begin{array}{l}S=4 \\
100 \% \\
R=0 \\
0 \%\end{array}$ & $\begin{array}{l}S=0 \\
0 \% \\
R=4 \\
100 \%\end{array}$ & $\begin{array}{l}S=0 \\
0 \% \\
R=4 \\
100 \%\end{array}$ & $\begin{array}{l}S=0 \\
0 \% \\
R=2 \\
100 \%\end{array}$ & $\begin{array}{l}S=2 \\
100 \% \\
R=0 \\
0 \%\end{array}$ \\
\hline $\begin{array}{l}\text { Ampicillin } \\
(\mathrm{n}=\mathbf{8 2}) \\
\mathrm{IS}=\mathbf{0}\end{array}$ & $\begin{array}{l}S=6 \\
(15.78 \%) \\
R=32 \\
\mathbf{8 4 . 2 \%}\end{array}$ & $\begin{array}{l}S=2 \\
(14.28 \%) \\
R=12 \\
\mathbf{8 5 . 7 1 \%}\end{array}$ & $\begin{array}{l}S=2 \\
(16.6 \%) \\
R=10 \\
83.33 \%\end{array}$ & $\begin{array}{l}S=2 \\
50 \% \\
R=2 \\
50 \%\end{array}$ & $\begin{array}{l}S=0 \\
(0 \%) \\
R=4 \\
100 \%\end{array}$ & $\begin{array}{l}S=0 \\
(0 \%) \\
R=4 \\
100 \%\end{array}$ & $\begin{array}{l}S=0 \\
(0 \%) \\
R=4 \\
100 \%\end{array}$ & $\begin{array}{l}S=0 \\
(0 \%) \\
R=2 \\
100 \%\end{array}$ \\
\hline $\begin{array}{l}\text { Amoxycillin } \\
(n=50) \\
I S=0\end{array}$ & $\begin{array}{l}S=2 \\
8.33 \% \\
R=22 \\
91.66 \%\end{array}$ & $\begin{array}{l}S=0 \\
0 \% \\
R=8 \\
100 \%\end{array}$ & $\begin{array}{l}S=2 \\
20 \% \\
R=8 \\
80 \%\end{array}$ & $\begin{array}{l}S=0 \\
(0 \%) \\
R=2 \\
100 \%\end{array}$ & $\begin{array}{l}S=0 \\
0 \% \\
R=2 \\
100 \%\end{array}$ & $\begin{array}{l}S=0 \\
0 \% \\
R=2 \\
100 \%\end{array}$ & $\begin{array}{l}S=0 \\
0 \% \\
R=2 \\
100 \%\end{array}$ & ND \\
\hline $\begin{array}{l}\text { Amox-clav } \\
(n=36) \\
\text { IS }=0\end{array}$ & $\begin{array}{l}S=0 \\
0 \% \\
R=18 \\
100 \%\end{array}$ & $\begin{array}{l}S=0 \\
0 \% \\
R=2 \\
100 \%\end{array}$ & $\begin{array}{l}S=0 \\
0 \% \\
R=6 \\
\mathbf{1 0 0 \%}\end{array}$ & $\begin{array}{l}S=4 \\
100 \% \\
R=0 \\
0 \%\end{array}$ & $\begin{array}{l}S=0 \\
(0 \%) \\
R=4 \\
100 \%\end{array}$ & $\begin{array}{l}S=0 \\
0 \% \\
R=4 \\
100 \%\end{array}$ & ND & ND \\
\hline
\end{tabular}


Table 4b: The Antibiotic Sensitivity of the Organisms Isolated in all the Cases.

\begin{tabular}{|c|c|c|c|c|c|c|c|c|}
\hline & \multicolumn{8}{|c|}{ Organisms (\% sensitive) } \\
\hline Drugs & 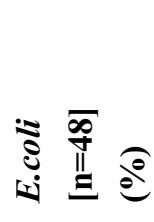 & 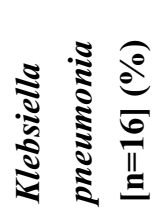 & 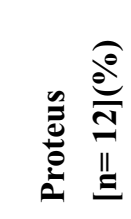 & 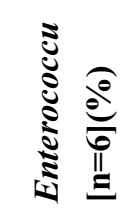 & 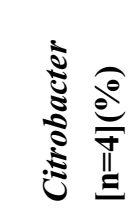 & 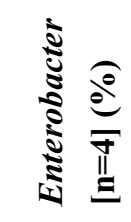 & 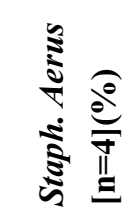 & 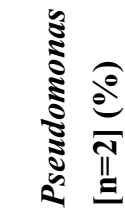 \\
\hline $\begin{array}{l}\text { Cephalhexin } \\
(\mathrm{n}=12) \\
\mathrm{IS}=\mathbf{0}\end{array}$ & $\begin{array}{l}S=2 \\
100 \% \\
R=0 \\
0 \%\end{array}$ & $\begin{array}{l}S=2 \\
\mathbf{1 0 0 \%} \\
R=0 \\
0 \%\end{array}$ & $\begin{array}{l}\mathrm{S}=2 \\
50 \% \\
\mathrm{R}=2 \\
50 \%\end{array}$ & ND & $\begin{array}{l}S=0 \\
0 \% \\
R=2 \\
100 \%\end{array}$ & ND & $\begin{array}{l}S=0 \\
0 \% \\
R=2 \\
100 \%\end{array}$ & ND \\
\hline $\begin{array}{l}\text { Cefotaxime } \\
(n=34) \\
\text { IS }=0\end{array}$ & $\begin{array}{l}\mathrm{S}=4 \\
22.22 \% \\
\mathrm{R}=14 \\
\mathbf{7 7 . 7 7 \%}\end{array}$ & $\begin{array}{l}S=2 \\
33.33 \% \\
R=4 \\
66.66 \%\end{array}$ & $\begin{array}{l}S=0 \\
0 \% \\
R=2 \\
100 \%\end{array}$ & ND & $\begin{array}{l}S=2 \\
50 \% \\
R=2 \\
50 \%\end{array}$ & $\begin{array}{l}S=0 \\
0 \% \\
R=4 \\
100 \%\end{array}$ & ND & ND \\
\hline $\begin{array}{l}\text { Ceftriaxone } \\
(n=12) \\
I S=0\end{array}$ & $\begin{array}{l}S=2 \\
33.33 \% \\
R=4 \\
66.66 \%\end{array}$ & ND & ND & ND & ND & $\begin{array}{l}S=0 \\
0 \% \\
R=2 \\
100 \%\end{array}$ & $\begin{array}{l}S=0 \\
0 \% \\
R=2 \\
\mathbf{1 0 0 \%}\end{array}$ & $\begin{array}{l}S=2 \\
100 \% \\
R=0 \\
0 \%\end{array}$ \\
\hline $\begin{array}{l}\text { Cephazolin } \\
(n=70)\end{array}$ & $\begin{array}{l}S=12 \\
31.57 \% \\
R=24 \\
\mathbf{6 3 . 1 5 \%} \\
\mathrm{IS}=2 \\
10.52 \%\end{array}$ & $\begin{array}{l}S=6 \\
75 \% \\
R=2 \\
25 \% \\
I S=0 \\
0 \%\end{array}$ & $\begin{array}{l}S=4 \\
33.33 \% \\
R=8 \\
66.66 \% \\
I S=0 \\
0 \%\end{array}$ & ND & $\begin{array}{l}S=0 \\
0 \% \\
R=4 \\
100 \% \\
I S=0 \\
0 \%\end{array}$ & $\begin{array}{l}S=0 \\
0 \% \\
R=4 \\
\mathbf{1 0 0} \% \\
I S=0 \\
0 \%\end{array}$ & $\begin{array}{l}S=0 \\
0 \% \\
R=2 \\
100 \% \\
I S=0 \\
0 \%\end{array}$ & $\begin{array}{l}S=0 \\
0 \% \\
R=2 \\
100 \% \\
I S=0 \\
0 \%\end{array}$ \\
\hline $\begin{array}{l}\text { Ceftazidime } \\
(\mathrm{n}=14) \\
\mathrm{IS}=\mathbf{0}\end{array}$ & $\begin{array}{l}S=2 \\
33.33 \% \\
R=4 \\
66.66 \%\end{array}$ & ND & $\begin{array}{l}S=0 \\
0 \% \\
R=2 \\
100 \%\end{array}$ & ND & $\begin{array}{l}S=0 \\
0 \% \\
R=2 \\
100 \%\end{array}$ & $\begin{array}{l}S=0 \\
0 \% \\
R=2 \\
100 \%\end{array}$ & ND & $\begin{array}{l}S=0 \\
0 \% \\
R=2 \\
100 \%\end{array}$ \\
\hline $\begin{array}{l}\text { Ofloxacin } \\
(n=12) \\
\text { IS }=0\end{array}$ & $\begin{array}{l}\mathrm{S}=2 \\
33.33 \% \\
\mathrm{R}=4 \\
\mathbf{6 6 . 6 6 \%}\end{array}$ & ND & ND & $\begin{array}{l}S=2 \\
100 \% \\
R=0 \\
0 \%\end{array}$ & ND & $\begin{array}{l}S=0 \\
0 \% \\
R=2 \\
100 \%\end{array}$ & $\begin{array}{l}S=0 \\
0 \% \\
R=2 \\
100 \%\end{array}$ & ND \\
\hline
\end{tabular}

$\mathbf{R}=$ Resistant

$\mathrm{S}=$ sensitive

IS=intermediate sensitive

ND=sensitivity not tested 
Table 4c: The Antibiotic Sensitivity of the Organisms Isolated in all the Cases.

\begin{tabular}{|c|c|c|c|c|c|c|c|c|}
\hline & \multicolumn{8}{|c|}{ Organisms (\% sensitive) } \\
\hline Drugs & 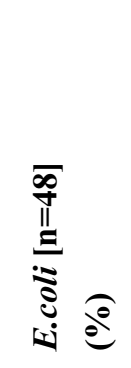 & 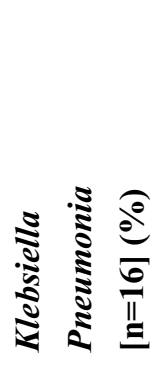 & 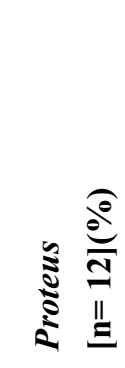 & 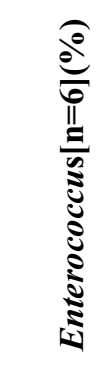 & 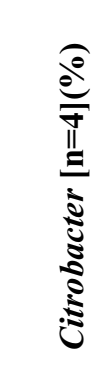 & 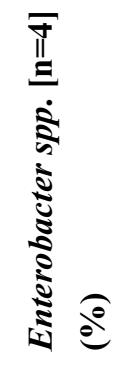 & 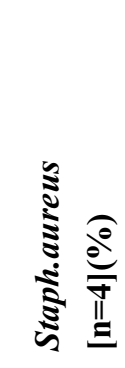 & 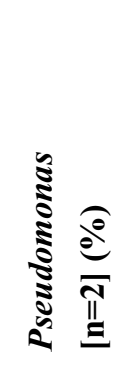 \\
\hline $\begin{array}{l}\text { Ciprofloxacin } \\
(n=42)\end{array}$ & $\begin{array}{l}S=12 \\
60 \% \\
R=6 \\
30 \% \\
I S=2 \\
10 \%\end{array}$ & $\begin{array}{l}S=4 \\
\mathbf{1 0 0} \% \\
R=0 \\
0 \% \\
I S=0 \\
0 \%\end{array}$ & $\begin{array}{l}S=4 \\
\mathbf{1 0 0} \% \\
\mathrm{R}=0 \\
0 \% \\
\mathrm{IS}=0 \\
0 \%\end{array}$ & $\begin{array}{l}S=4 \\
\mathbf{1 0 0} \% \\
R=0 \\
0 \% \\
I S=0 \\
0 \%\end{array}$ & $\begin{array}{l}S=2 \\
\mathbf{1 0 0} \% \\
R=0 \\
0 \% \\
I S=0 \\
0 \%\end{array}$ & $\begin{array}{l}S=4 \\
\mathbf{1 0 0} \% \\
R=0 \\
0 \% \\
I S=0 \\
0 \%\end{array}$ & $\begin{array}{l}S=2 \\
\mathbf{1 0 0} \% \\
R=0 \\
0 \% \\
I S=0 \\
0 \%\end{array}$ & $\begin{array}{l}S=2 \\
\mathbf{1 0 0} \% \\
R=0 \\
0 \% \\
\text { IS }=0 \\
0 \%\end{array}$ \\
\hline $\begin{array}{l}\text { Norfloxacin } \\
(\mathrm{n}=74) \\
\mathrm{IS}=\mathbf{0}\end{array}$ & $\begin{array}{l}S=28 \\
\mathbf{7 3 . 6 8 \%} \\
R=10 \\
26.31 \%\end{array}$ & $\begin{array}{l}S=10 \\
\mathbf{8 3 . 3 3 \%} \\
R=2 \\
16.66 \%\end{array}$ & $\begin{array}{l}S=12 \\
100 \% \\
R=0 \\
0 \%\end{array}$ & $\begin{array}{l}S=2 \\
100 \% \\
R=0 \\
0 \%\end{array}$ & $\begin{array}{l}S=2 \\
50 \% \\
R=2 \\
50 \%\end{array}$ & $\begin{array}{l}S=2 \\
100 \% \\
R=0 \\
0 \%\end{array}$ & $\begin{array}{l}S=2 \\
100 \% \\
R=0 \\
0 \%\end{array}$ & $\begin{array}{l}S=0 \\
0 \% \\
R=2 \\
100 \%\end{array}$ \\
\hline $\begin{array}{l}\text { Nalidixic acid } \\
(\mathrm{n}=32) \\
\mathrm{IS}=\mathbf{0}\end{array}$ & $\begin{array}{l}S=6 \\
27.27 \% \\
R=16 \\
72.72 \%\end{array}$ & $\begin{array}{l}S=2 \\
100 \% \\
R=0 \\
0 \%\end{array}$ & $\begin{array}{l}S=4 \\
100 \% \\
R=0 \\
0 \%\end{array}$ & ND & ND & $\begin{array}{l}S=2 \\
100 \% \\
R=0 \\
0 \%\end{array}$ & ND & $\begin{array}{l}S=0 \\
0 \% \\
R=2 \\
100 \%\end{array}$ \\
\hline $\begin{array}{l}\text { Nitrofurantoin } \\
(n=54) \\
\text { IS }=0\end{array}$ & $\begin{array}{l}S=30 \\
100 \% \\
R=0 \\
0 \%\end{array}$ & $\begin{array}{l}S=4 \\
50 \% \\
R=4 \\
50 \%\end{array}$ & $\begin{array}{l}S=8 \\
100 \% \\
R=0 \\
0 \%\end{array}$ & $\begin{array}{l}S=2 \\
100 \% \\
R=0 \\
0 \%\end{array}$ & $\begin{array}{l}S=2 \\
100 \% \\
R=0 \\
0 \%\end{array}$ & $\begin{array}{l}S=0 \\
0 \% \\
R=2 \\
100 \%\end{array}$ & ND & $\begin{array}{l}S=2 \\
\mathbf{1 0 0} \% \\
R=0 \\
0 \%\end{array}$ \\
\hline $\begin{array}{l}\text { Co-trimoxazole } \\
(n=66) \\
\text { IS }=0\end{array}$ & $\begin{array}{l}\mathrm{S}=16 \\
44.44 \% \\
\mathrm{R}=20 \\
55.55 \%\end{array}$ & $\begin{array}{l}S=6 \\
50 \% \\
R=6 \\
50 \%\end{array}$ & $\begin{array}{l}S=10 \\
100 \% \\
R=0 \\
0 \%\end{array}$ & ND & $\begin{array}{l}S=2 \\
100 \% \\
R=0 \\
0 \%\end{array}$ & $\begin{array}{l}S=2 \\
100 \% \\
R=0 \\
0 \%\end{array}$ & $\begin{array}{l}S=2 \\
50 \% \\
R=2 \\
50 \%\end{array}$ & ND \\
\hline $\begin{array}{l}\text { Vancomycin } \\
(\mathrm{n}=14) \\
\mathrm{IS}=\mathbf{0} \\
\mathrm{R}=\mathbf{0}\end{array}$ & $\begin{array}{l}S=2 \\
100 \%\end{array}$ & $\begin{array}{l}S=2 \\
100 \%\end{array}$ & ND & $\begin{array}{l}S=6 \\
100 \%\end{array}$ & ND & ND & $\begin{array}{l}S=4 \\
100 \%\end{array}$ & ND \\
\hline
\end{tabular}

$R=$ Resistant $\quad$ S=sensitive $\quad$ IS=intermediate sensitive $\quad$ ND=sensitivity not tested 


\section{Discussion}

Urinary tract infections are relatively common in children and are a common cause of fever with absence of other urinary symptoms. ${ }^{1}$ The exact rates of UTI are unknown because as many as $40 \%$ of infections in infants and children are asymptomatic. It has been estimated that approximately $3 \%$ of girls and $1 \%$ of boys have symptomatic UTI before the age of 11 years. ${ }^{2}$ Yet other studies state that up to 7 percent of girls and 2 percent of boys will have a symptomatic, culture-confirmed urinary tract infection by six years of age. ${ }^{3}$

In this study $55(32.7 \%)$ males and $113(67.2 \%)$ females with male to female ratio 1:2 were diagnosed to have UTI. Amongst these children, $86.9 \%$ presented with febrile UTI and the remaining $13.1 \%$ had other symptoms. Urinary symptoms like dysuria (23.8\%), burning urine(15.4\%), increased frequency $(13 \%)$, haematuria (10\%), oliguria $(7.1 \%)$, bed wetting $(2.38 \%)$ were also noted. Other symptoms were pain abdomen (46.4\%), vomiting (39.28\%), chills and rigors (17.8\%), loose stool(14.28\%), febrile seizures (13\%), concomitant respiratory tract infections $(13 \%) .7 .1 \%$ cases presented with facial puffiness, constipation, hepatosplenomegaly each. Vaginal discharge was present in $(5.95 \%)$ cases. Other presentations were irritability, failure to thrive, decreased appetite, phimosis. Fever and nonspecific symptoms $^{3}$, cloudy urine ${ }^{3}$, failure to thrive in infant ${ }^{3}$, enueresis $^{4}$, diarrhea ${ }^{5}$ in UTI was also noted by some other authors.

The first and most critical step in establishing the diagnosis of UTI in infants and young children is the method by which urine is collected. In the young patient care must be taken to prepare carefully the perineum and periurethral area for placement of the sterile plastic receptacle for collection of urine. In the infant the surest way to obtain urine for culture aseptically is by percutaneous suprapubic aspiration. Older children and adolescents can be instructed to collect a midstream urine specimen after proper cleansing of the urethral area. These steps were strictly followed for collection of urine in our study. The presence of $10^{5}$ organisms or more per $\mathrm{ml}$ of urine is diagnostic of UTI. As few as $5 \times 10^{2}$ to $10^{4}$ organisms $/ \mathrm{ml}$ have been etiologically associated with the frequency/dysuria syndrome in women. ${ }^{6}$ In this study only the ones showing colony $\geq 10^{5}$ and the organisms from suprapubic puncture was considered culture positive cases. If $10^{3}$ to $10^{5}$ colony forming units of a single genus and species per $\mathrm{ml}$ are recovered from two successive urine cultures of a child, a diagnosis of UTI should be made ${ }^{6}$. In our context such cases were not included in our study as it was difficult to call the patient back for repeat urine culture though they were empirically treated as suspected UTI. The suprapubic bladder aspiration or by catherization may contain fewer than $10^{5}$ organisms $/ \mathrm{ml}$ because the bacteria have not had sufficient time to multiply before removal of urine from the bladder. ${ }^{6}$
In this study $96(57 \%)$ resulted a positive culture in urine with significant colony count of $\geq 10^{5}$. The rest 72 $(42.9 \%)$ were either culture negative or they had colony count $<10^{5}$. (Fig 1) Like other studies, ${ }^{\mathbf{7 , 8 9}}$ E.coli (50\%) was the commonest pathogen isolated in our study too. But unlike our study the percentage was higher (80$86 \%)^{7,8,9}$ in their studies. Our study was consistent with the study conducted in adults earlier in our hospital where the pathogens isolated were E.coli (59.4\%), Klebsiella $(15.7 \%)$ and Enterococcus faecalis $(8.1 \%) .{ }^{10}$ Our distribution of pathogens were E.coli (50\%), Klebsiella pneumoniae (16.66\%), Proteus vulgaris (12.50\%), Enterococcus fecalis (6.25\%), Citrobacter, (4.10\%) Enterobacter (4.10\%), Staphylococcus aureus (4.10\%) and Pseudomonas aeruginosa (2.08\%). Similar findings were also reported by a study in Sousse, Tunisia where the pathogens were E. coli(71\%), Klebsiella pneumoniae (10\%), Proteus mirabilis (8\%), Staphylococcus (1.6\%), Pseudomonas aeruginosa (1\%) and others $(2 \%) .{ }^{11}$ Yet in another two studies the findings were consistent with ours where the pathogens were E. coli (57.3\%), Proteus spp. (15.6\%) and Pseudomonas aeruginosa (9.1\%) ${ }^{12}$ in one study and Escherichia coli $(57.5 \%)$, Klebsiella pneumoniae (12.4\%), Enterococcus spp.(6.6\%), Proteus mirabilis (5.4\%), Pseudomonas aeruginosa (2.9\%), Citrobacter spp. (2.7\%), Staphylococcus aureus (2.2\%), Enterobacter cloacae $(1.9 \%)^{13}$ in another study.

In our study Amikacin had the highest sensitivity (96\%) among the Aminoglycosides even though Enterococcus and Pseudomonas were resistant to the same (Table 4a). Netilmycin and Gentamycin had sensitivity of $70.8 \%$ and $64.86 \%$ respectively. Sensitivity with Nitrofurantoin $(88.88 \%)$, Norfloxacin (78.3\%), Ciprofloxacin (80.9\%) were also appreciable. Sensitivity for Vancomycin (100\%), Cephalexin (50\%), Ofloxacin (33.33\%) and Ceftriaxone $(33.33 \%)$ was tested only in 12- 14 cases. Vancomycin was found to be $100 \%$ sensitive to Enterococcus, Staphylococcus, E.coli and Klebsiella. Highest degree of resistance was noticed with Amoxicillin (92\%) Ampicillin (85.36\%), Ceftazidime (85.71\%), Amoxy-clav (83.33\%), Cefotaxime (76.47\%), Cefazolin (62.85\%), Ofloxacin $(66.66 \%)$ and Ceftriaxone $(66.66 \%)$.

The sensitivity pattern of various organisms were also studied (Table 4a,4b,4c). E. coli susceptibility to antibiotics was characterised by the high resistance to Ampicillin (84.2\%), Amoxicillin (91.66\%\%) and Amoxy-clav (100\%). The resistance percentage to Cephalosporins was also high $(63 \%-77 \%)$. It had a good sensitivity to Aminoglycosides (69.2\%-100\%), Fluroquinolones $(33.3 \%-73.7 \%)$ and to Nitrofurantoin $(100 \%)$. This pattern was also observed in the study conducted in Tunisia where the microorganism distribution was same as ours. In the former study ${ }^{11}$ the resistance pattern was to Amoxicillin (60\%), to Amoxy- clav (54\%), Aminoglycosides (1\%) and to Nitrofurantoin (1\%). Unlike our study resistance to Cotrimoxazole was observed in only $40 \%$ as compared 
to our $55.55 \%$. Cephalosporin resistance was $1.5 \%$ in their study while ours was between $63 \%-77 \%{ }^{11}$. The sensitivity pattern of Klebsiella and Proteus were similar a with few minor differences such as Proteus showing higher sensitivity pattern towards Norfloxacin $(100 \%)$, Nitrofurantoin $(100 \%)$ and Co-trimoxazole $(100 \%)$ as compared to Klebsiella showing sensitivity to Norfloxacin (83.33\%), Nitrofurantoin $(50 \%)$ and Co-trimoxazole(50\%). High resistance rates among Klebsiella pneumoniae strains towards to Amoxicillin and Amoxy-clav and Cefotaxime, respectively of 63,63 and 39\% were noticed. ${ }^{11}$ This was $100 \%, 100 \%, 66.66 \%$ respectively in our study. The resistance percentages to Amikacin and Cotrimoxazole were respectively $17 \%$ and $65 \%$ in theirs and $0 \%$ and $50 \%$ in ours.

Resistance to third-generation Cephalosporins is caused by the acquisition of plasmids containing genes that encode for extended-spectrum beta-lactamases (ESBLs), and these plasmids often carry other resistance genes as well. ${ }^{14}$ ESBL-producing Klebsiella pneumoniae and Escherichia coli are now relatively common in healthcare settings and often exhibit multidrug resistance. Resistance of Enterobacter species to third-generation cephalosporins is most typically caused by overproduction of AmpC beta-lactamases, and treatment with third-generation cephalosporins may select for AmpC-overproducing mutants. Some Enterobacter cloacae strains are now ESBL and AmpC producers, conferring resistance to both thirdand fourth-generation Cephalosporins. Quinolone resistance in Enterobacteriaceae is usually the result of chromosomal mutations leading to alterations in target enzymes or drug accumulation. More recently, however, plasmid-mediated Quinolone resistance has been reported in Klebsiella pneumoniae and E coli, associated with acquisition of the qnr gene. ${ }^{14}$ Seventy percent (70\%) of Proteus strains were resistant to Amoxicillin, $63 \%$ of them remained susceptible to Amoxy-clav. No resistance was shown to Amikacin against 31\% towards Cotrimoxazole. ${ }^{11}$ In our study resistance was $80 \%$ with Amoxicillin, 100\% with Amoxy-clav, 0\% to Amikacin and $0 \%$ with Co-trimoxazole.

The resistance pattern found for above organisms in some other studies were for Ampicillin (100\%), Erythromycin (100\%), Penicillin (100\%), Co-trimoxazole (60\%), Tetracycline (60\%) and Chloramphenicol $(60 \%)^{15}$. In another study the resistance pattern for Ampicillin was $(83.5 \%)$ and for Co-trimoxazole $(76.7 \%)^{16}$, This was $37.7 \%$ for Ampicillin, $921.3 \%$ for SMX/TMP, $5.5 \%$ for Ciprofloxacin, $5.1 \%$ for Levofloxacin and $1.1 \%$ for Nitrofurantoin ${ }^{13}$.The resistance was $74.2 \%$ for Ampicillin and $61.3 \%$ for Co-trimoxazole in another study. ${ }^{17}$ Enterobacter, Citrobacter, Staphylococcus and Pseudomonas were the less commonly isolated organisms in our study, They were highly resistant (80-100\% ) to Ampicillin, Amoxycillin, Amoxyclav and Cephalosporins. They were $100 \%$ sensitive to Amikacin and Netlimycin but were $100 \%$ resistant to Gentamycin except for Staphylococcus which was $100 \%$ resistant to Netlimycin and Pseudomonas was $100 \%$ sensitive to Gentamycin. Staphylococcus susceptibitlity to Fluoroquinolones was seen to be $100 \%$ sensitivite to Ciprofloxacin and Norfloxacin but $100 \%$ resistant to Ofloxacin. Unlike other organisms Enterococcus showed 100\% resistance to Amikacin but was $100 \%$ sensitive to Gentamycin. Compared to other organisms its resistance was lower with Amoxyclav $(0 \%)$ and Ofloxacin $(0 \%)$. It showed no resistance to Fluoroquinolones, Nitrofurantoin and Vancomycin. Vancomycin showed no resistance but the sensitivity for Vancomycin was tested only in $14.5 \%$ of the cases. In a study by Nowakowska et al, only $9.4 \%$ of isolated Enterococcus spp. were high-level Aminoglycoside resistant (HLAR) strains. No Vancomycin-resistant Enterococcus spp. were isolated. ${ }^{18}$

\section{Conclusion}

As UTI is a significant problem in the children and still continues to be a major threat for morbidity and mortality in subtropical parts of the world, larger scale studies must be carried out at regular intervals in order to identify the changing trend in the pathogenic organisms and update on its changing antibiotic susceptibility. Based on the above sensitivity pattern we recommend empirical use of Amikacin and Netlimycin for hospitalized patients whereas Nitrofurantoin, Norfloxacin and Ciprofloxacin for outpatient is appropriate in children with UTI. As Vancomycin showed $100 \%$ sensitivity to some of the not so common organisms, it could be kept as a reserve drug for resistant cases.

\section{References}

1. Handel LN, Caldamone AA. Urinary Tract Infections in the Pediatric Population. J Med Liban 2004 Oct-Dec;52(4):194-201.

2. Winberg J. Epidemiology of Symptomatic Urinary Tract Infections in Childhood. Acta Paediatr Scand Suppl 1974:252.

3. Alper BS, Curry SH. Urinary Tract Infection in Children. Am Fam Physician. 2005 Dec 15; 72(12):2483-8.

4. Nevéus T. The Evaluation and Treatment of Therapy-Resistant Enuresis: A Review: Ups J Med Sci. 2006;111(1):61-71.

5. Fallahzadeh MH, Ghane F. Urinary Tract Infection in Infants and Children with Diarrhoea. East Mediterr Health J. 2006 Sep;12(5):690-4.

6. Stamm WE, Counts GW, Running KR, et al. Diagnosis of Coliform Infection in Acutely Dysuric Women. N Engl J Med 1982; 307:463-8. 
7. Hernández-Porras M, Salmerón-Arteaga G, Medina-Santillán R. Microbial Resistance to Antibiotics used to Treat Urinary Tract Infections in Mexican Children. Proc West Pharmacol Soc. 2004;47:120-1.

8. Lizama C M, Luco I M, Reichhard T C, Hirsch B T. Urinary Tract Infection in a Pediatrics Emergency Department: Frequency and Clinical Parameters. Rev Chilena Infectol. 2005 Sep;22(3):235-41.

9. Sobczyk D, Krynicki T, Blumczyński A, Zaniew M, Kroll P, Siwińska A, Zachwieja J. New, Successful Treatment of Urinary Tract Infection Caused by Pseudomonas Aeruginosa. Przegl Lek. 2006; 63 Suppl 3:140-1

10. Das RN, Chandrashekhar TS, Joshi HS, Gurung M, Shrestha N, Shivananda PG Frequency and Susceptibility Profile of Pathogens Causing Urinary Tract Infections at a tertiary Care Hospital In Western Nepal. Singapore Med J. 2006 Apr;47(4):281-5.

11. Bouallègue $\mathrm{O}$, Saidani $\mathrm{M}$, Ben Mohamed S, Mzoughi R. Bacteriologic Features of Urinary Tract Infections in Children in the Sousse Area, Tunisia. Tunis Med. 2004 Aug;82(8):742-6.

12. Zajaczkowska M, Zinkiewicz Z, Bieniaś B, Piechuta L, Szajner-Milart I, Majewski M. Recurrent Urinary Tract Infections--Uropathogen Incidence and Drug Sensitivity. Ann Univ Mariae Curie Sklodowska [Med]. 2004;59(2):275-83.

13. Zhanel GG, Hisanaga TL, Laing NM, DeCorby MR, Nichol KA, Palatnik LP, Johnson J, Noreddin A, Harding GK, Nicolle LE, Hoban DJ; NAUTICA Group. Antibiotic Resistance In Outpatient Urinary Isolates: Final Results from the North American Urinary Tract Infection Collaborative Alliance (NAUTICA). Int $J$ Antimicrob Agents. 2005 Nov;26(5):380-8.
14. Paterson DL. Resistance in Gram-Negative Bacteria: Enterobacteriaceae. Am J Med.2006 Jun;119(6 Suppl 1):S20-8; discussion S62-70

15. Tessema B, Kassu A, Mulu A, Yismaw G. Pridominant Isolates of Urinary Tract Pathogens and their Antimicrobial Susceptiblity Patterns in Gondar University Teaching Hospital, Northwest Ethiopia. Ethiop Med J. 2007 Jan;45(1):61-7

16. Hernández-Porras $M$, Salmerón-Arteaga G, Medina-Santillán R. Microbial Resistance to Antibiotics used to Treat Urinary Tract Infections in Mexican Children. Proc West Pharmacol Soc. 2004;47:120-1.

17. Yüksel S, Oztürk B, Kavaz A, Ozçakar ZB, Acar B, Güriz H, Aysev D, Ekim M, Yalçinkaya F. Antibiotic Resistance of Urinary Tract Pathogens and Evaluation of Empirical Treatment in Turkish Children with Urinary Tract Infections. Int $J$ Antimicrob Agents. 2006 Nov;28(5):413-6. Epub 2006 Sep 26.

18. Nowakowska M, Rogala-Zawada D, Wiechuła B, Rudy M, Radosz-Komoniewska H, Zientara M. Urinary Tract Infections in Children--Etiologic Agents and Susceptibility to Antibiotics. Int $J$ Antimicrob Agents. 2006 Nov;28(5):413-6. Epub 2006 Sep 26. 